\title{
China's Demographic Challenges from a Global Perspective
}

Zhongwei Zhao

\section{Introduction}

One of the most significant events in recent history has been the worldwide demographic transition. The role of this transition 'in the creation of the modern world' has been so important that some scholars have argued that unless the demographic transition is put at centre-stage, the modern process of development cannot be understood (Dyson 2010:viii). Population changes taking place in China have also been closely related to its historical development and will greatly influence the country's future. This chapter describes China's demographic transition in a global perspective, and examines the major demographic trends and challenges that it will face in the next few decades.

\section{Demographic transition in China and the world}

\section{Demographic changes before the mid-twentieth century}

Europe was the leader of the demographic transition, with long-term mortality decline already beginning in a number of countries in the early nineteenth century. In the United Kingdom, France and Sweden, for example, life expectancies at birth increased from 37.3, 33.9 and 36.5 years in 1800-09 to 69.2, 66.5 and 71.3 years in 1950, respectively. While it started slightly later, fertility decline was also on the way about the mid-nineteenth century. Complete family size remained large for those born about 1800, with close to five children per woman; but lifetime fertility fell to about two children for those born about a century later in England and Wales, France and Sweden (Livi-Bacci 2007). According to the United Nations, Europe, North America, Australia, New Zealand and Japan had largely completed the classic demographic transition by the early 1950s, with life expectancy at birth reaching 66 years and the total fertility rate (TFR) declining to about three children per woman (UN 2009). In the early stage of demographic transition, the decrease of both mortality and fertility was relatively slow and took a long time to accomplish. This gave enough time for both society and government to respond to these changes and to prepare for their consequences.

In contrast, in other parts of the world, demographic transition started later. Mortality and fertility remained high in many countries in Africa, Asia and Latin America until the end of World War II. According to the United Nations, life expectancy for these countries (excluding China) was about 41 years and their TFR was more than six children per woman in the early 1950s (UN 2009). 
China was a typical country of this kind. According to a survey conducted among Chinese peasants in around 1930, their life expectancy at birth was about only 25 years and the TFR was about 5.5 (Barclay et al. 1976). Despite the improvement in lowering mortality in some Chinese cities during the first half of the twentieth century, the life expectancy for China's national population was likely to have been lower than 35 years and TFR was between five and six children in the late 1940s (Campbell 2001; Zhao 1997).

One of the most significant impacts of the pre-transitional demographic regime on the society and economic development was the great waste of human resources, which contributed directly to the slow economic growth of the time. Under the high mortality recorded among the Chinese peasant population about 1930, for example, to ensure that one son would survive to a marriageable age and continue the family line, each couple would need to have an average of at least five births, because more than 40 per cent of children did not survive to age fifteen. Accordingly, women had to spend most of their reproductive years in pregnancy, giving birth and taking care of children. But a large part of such effort and investment would bring about no return or be wasted completely because of high mortality. It is, therefore, not a surprise that low economic growth was often found in the pre-transitional society.

\section{Worldwide demographic transition in the second half of the twentieth century}

As shown in Figures 16.1-4, mortality and fertility have continued to decline in the industrialised world since the end of World War II. By the end of the twentieth century, life expectancy at birth rose from about 66 to about 75 years, and the TFR fell from nearly 3 to 1.6 children. As a result of these changes and the impact of international migration, the population size of the world's more industrialised countries increased from 812 million in 1950 to 1.195 billion in 2000. The age structure became older, with the proportion of people aged 65 and over growing from about 8 per cent to more than 14 per cent of the total population. Because of the fertility decline, however, the total dependency ratio, which is measured by the sum of the population aged $0-14$ and that aged 65 and over (or dependents) to the population aged 15-64 (or working-age population) and presented as the number of dependents per 100 persons of working age, was maintained at a comparatively low level in these countries. After fluctuating about 55 for a quarter of a century (1950-75), it fell steadily to 49 in the year 2000 (UN 2009). 
Figure 16.1 Changes in life expectancy at birth, 1950-2010

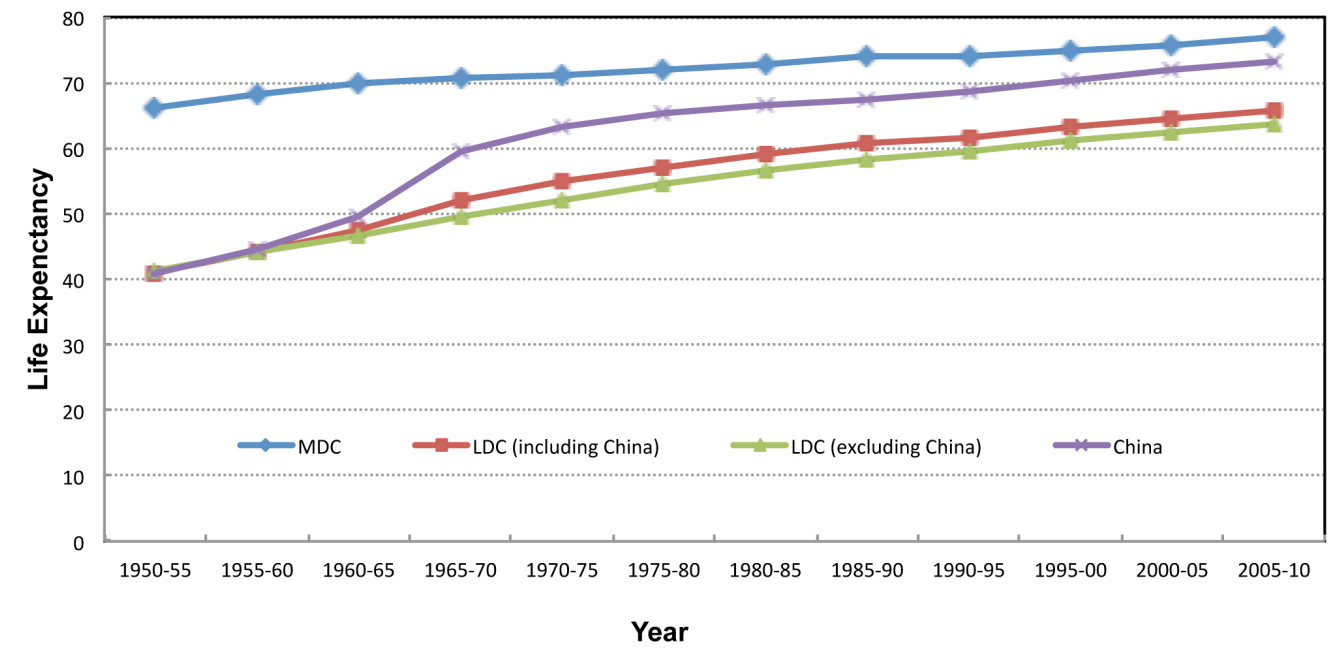

Source: UN (2009).

Figure 16.2 Changes in the total fertility rate, 1950-2010

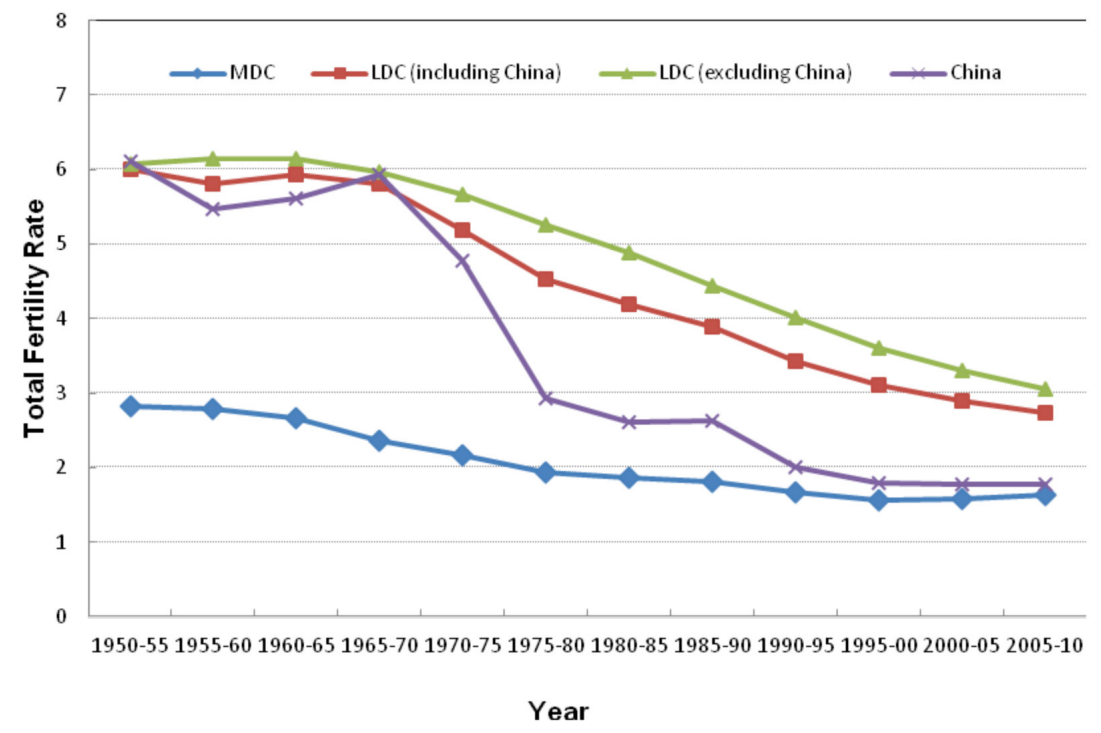

Source: UN (2009). 
Figure 16.3 Changes in the dependency ratio, 1950-2010

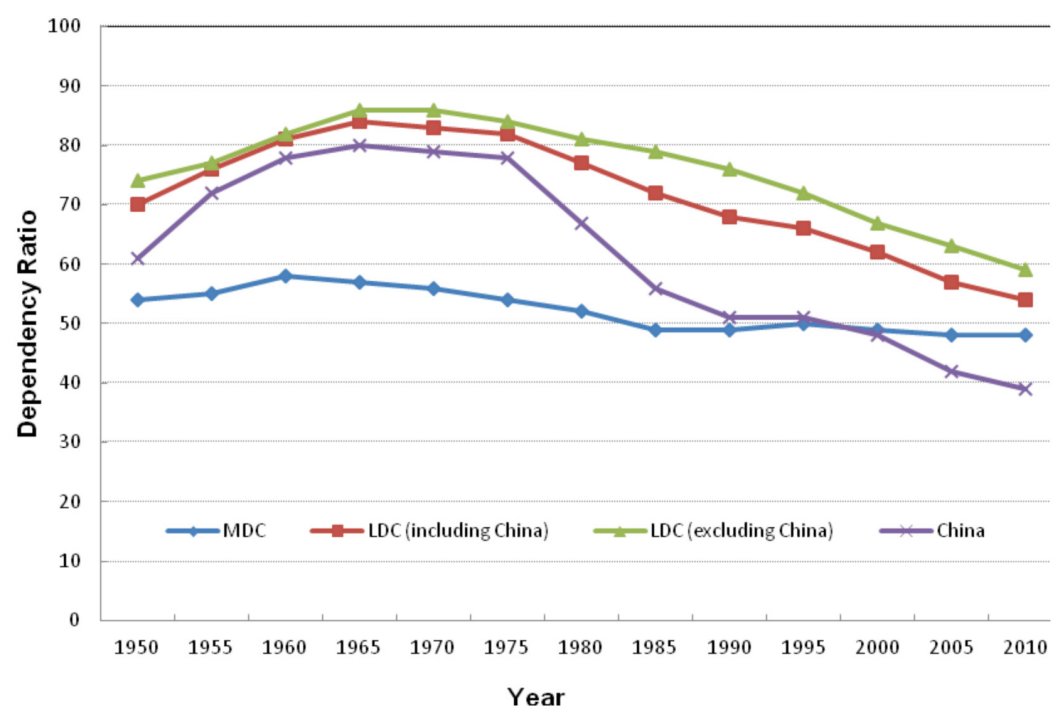

Source: UN (2009).

Figure 16.4 Changes in the proportion of people over age sixty-five, 1950-2010

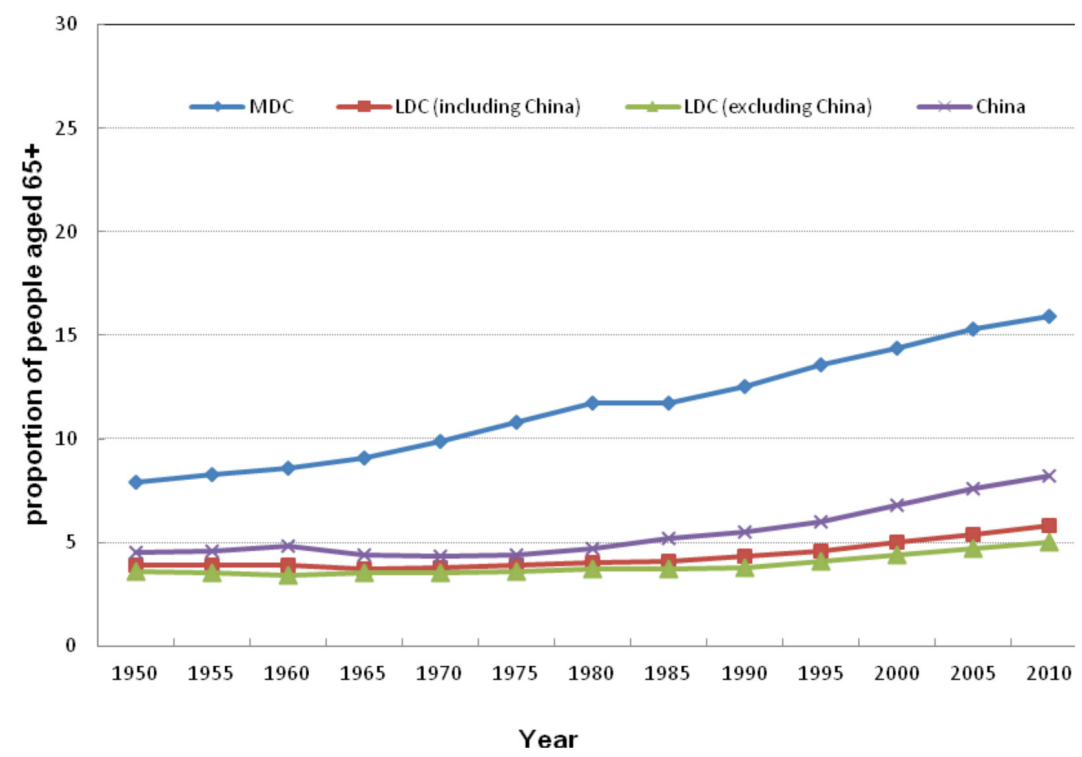

Source: UN (2009).

In less developed countries (excluding China), demographic transition began, or started to accelerate, after World War II. In the next half-century, life expectancy rose from 41 to 61 years and the TFR fell from 6.1 to 3.6 children per woman. There were, of course, great variations in the speed and magnitude of such transitions among these countries. 
The most rapid mortality and fertility declines took place in East Asia, while the least developed countries fell far behind, with a life expectancy of 52 years and a TFR of about 5 children per woman at the end of the twentieth century. From 1950 to 2000, the population size of less developed countries (excluding China) increased from 1.2 to 3.6 billion (from 1.7 to 4.9 billion, if China is included). Because of their rapid population growth, these countries also had a markedly higher dependency ratio than more industrialised countries - greater than 70 in most of the years. This was caused primarily by their high fertility, which contributes directly to increases in the child dependency ratio. For the same reason, age structures in many less developed countries either remained young or became younger in the second half of the twentieth century (UN 2009). The comparatively high dependency ratio has been a major demographic contributor to the slow development in many less developed countries.

\section{China's demographic transition and its recent economic growth}

Demographic changes taking place in China in the second half of the twentieth century were broadly similar to those observed in other less developed countries, but with greater speed and magnitude.

China witnessed a rapid improvement in mortality in the 1950s and 1960s except during the great famine. According to estimates made by Banister, life expectancy at birth reached about 50 years in 1957, 61 in 1970, and 65 in 1981, which are reasonably close to those calculated by the United Nations and displayed in Figure 16.1. China's official data suggest that mortality decline was even faster during this period (Banister 1987; Huang and Liu 1995). ${ }^{1}$ These experiences, along with those observed in Sri Lanka, Costa Rica and some other populations, have been widely seen as a great success in lowering mortality in poor countries (Caldwell 1986:171). Since the early 1980s, China's mortality has declined further. Life expectancy at birth for the national population has now reached 74 years (Ren et al. 2004; UN 2009; Zhao and Guo 2007). While the speed of recent mortality decline has been slower than that recorded earlier, it is still remarkable in comparison with the experience of some former Soviet republics and Eastern European countries where mortality stopped declining or even increased during their recent reconstruction (Meslé 2004).

China's national fertility level remained high in the 1950s and 1960s, although a nontrivial fertility decline was already observed in some urban populations at the time (Lavely and Freedman 1990). This and the falling mortality led to rapid population growth. Facing increasing population pressure, the Chinese Government launched an unprecedented familyplanning campaign in the early 1970s, which played an important part in bringing the fertility rate down to the level of below replacement. While China's TFR was still about six children at the beginning of the 1970s, it fell rapidly to about 2.5 in 1980. In the 1980s, China's fertility decline seemed to have stopped and the TFR fluctuated between 2.3 and 2.9 (Yao 1995). This, however, was followed by further fertility reduction in the 1990s. China's TFR had fallen to below replacement in 1991 and further declined to less than 1.6 in 2000. Many studies have suggested that China's fertility has remained that low or has fallen even lower since, which has been supported by recently published 2010 census preliminary results (Cai 2008, 2010; Guo and Chen 2007; Retherford et al. 2005; Scharping 2005; Zhang and Zhao 2006; Zhao and Guo 2010; Zhao and Zhang 2010; Zheng et al. 2009). They differ notably from those estimated by the United Nations in recent years, as shown in Figure 16.2. ${ }^{2}$ 
China's mortality and fertility decline opened a 'demographic window' - like other countries experiencing similar demographic changes. According to the United Nations' 2008 medium variant population projection, China's total dependency ratio was close to 80 in the early 1970s, but it has been falling since and has now reached 39, as indicated by Figure 16.3 (UN 2009). ${ }^{3}$ This favourable age structure, which provides auspicious demographic conditions for economic development, has been widely seen as the first demographic dividend. From a demographic point of view, it seems rather natural that China's spectacular economic development has taken place during this period.

\section{China's major demographic challenges in a global perspective in the early twenty-first century}

\section{The demographic map of the world at the beginning of the twenty-first century}

Worldwide mortality and fertility decline in recent decades has brought about many significant changes. If we use the United Nations' classification of more developed and less developed countries, it is evident that the demographic map of the world now differs greatly from half a century ago.

In the early 1950s, nearly one-third of the world population lived in more developed countries and the other two-thirds in less developed countries. In 2010, the population of more developed countries declined to less than one-fifth of the world total. The population of less developed countries accounted for more than four-fifths, which itself was more than twice the world population of 1950. These trends will continue in the next 40 years.

Comparing with half a century ago, the gap between more developed and less developed countries in their mortality and fertility has narrowed in general, as shown in Figures 16.1 and 16.2. In 2005-10, life expectancy at birth in less developed countries reached 66 years. This was about 85 per cent of that achieved in more developed countries - a marked increase from the 62 per cent recorded in 1950-55. Also in 2005-10, the TFR was 3.3 in less developed countries and 1.6 in more developed countries; this gap was also smaller than that in 1950-55. Although there are some uncertainties, further convergences in mortality and fertility are likely to take place in the two groups of countries in the next four decades.

During the past half-century, total dependency ratios declined in both more developed and less developed countries. As Figures 16.3 and 16.4 show, although the magnitude of such reduction is more noticeable in less developed countries, their dependency ratio is still higher than that in more developed countries. This is attributed mainly to their high child dependency ratios. In both more developed and less developed countries, the age structure has become older. The median age of the population has increased from 29 years in 1950 to the current 40 years in more developed countries and from 22 to 27 years in less developed countries (UN 2009).

In the next 40 years, more developed countries are likely to experience the following demographic changes. Their mortality will continue to fall and fertility could fluctuate at a relatively low level. As a result, their total population will be relatively stable and 
close to 1.3 billion. But their share in the world population will further shrink - to less than 14 per cent. Demographically, the influence of countries currently regarded as 'more developed' will be greatly reduced. Moreover, as shown in Figures 16.5 and 16.6, the age structure of their populations will become much older, with the proportion of those aged 65 and over increasing from the current 15.9 per cent to 26.2 per cent. Driven by this change, their total dependency ratios will rise markedly - from 48 currently to 71 in 2050. Because of that and labour shortages, which are partly a result of low fertility, immigration is likely to further increase in some countries.

Figure 16.5 Changes in the dependency ratio, 2000-50

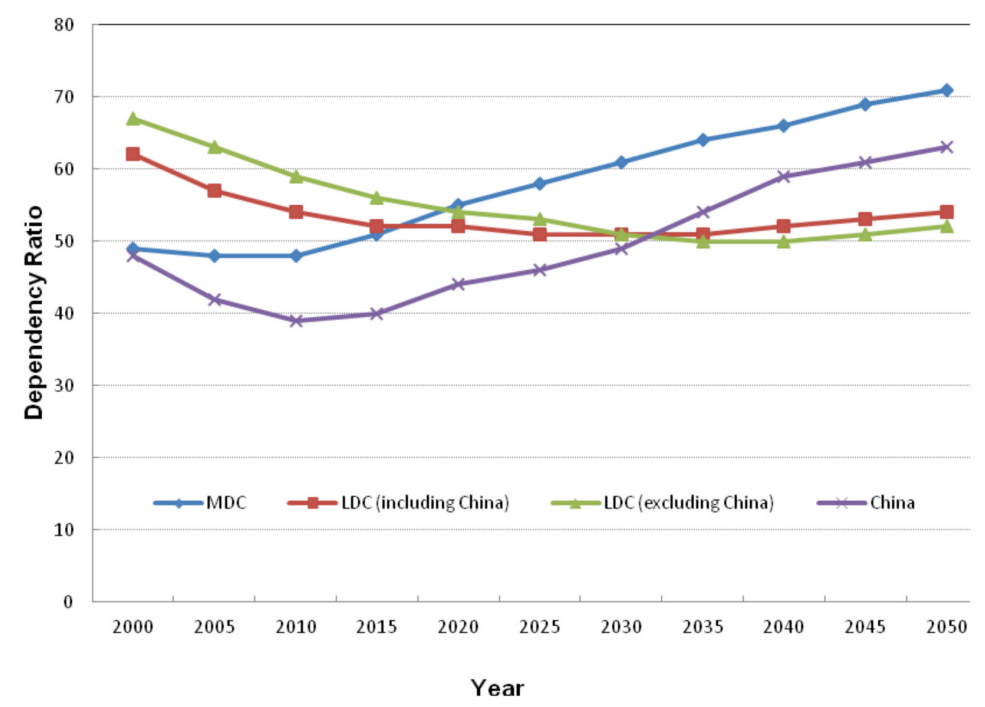

Source: UN (2009).

Figure 16.6 Changes in the proportion of people over age sixty-five, 2000-50

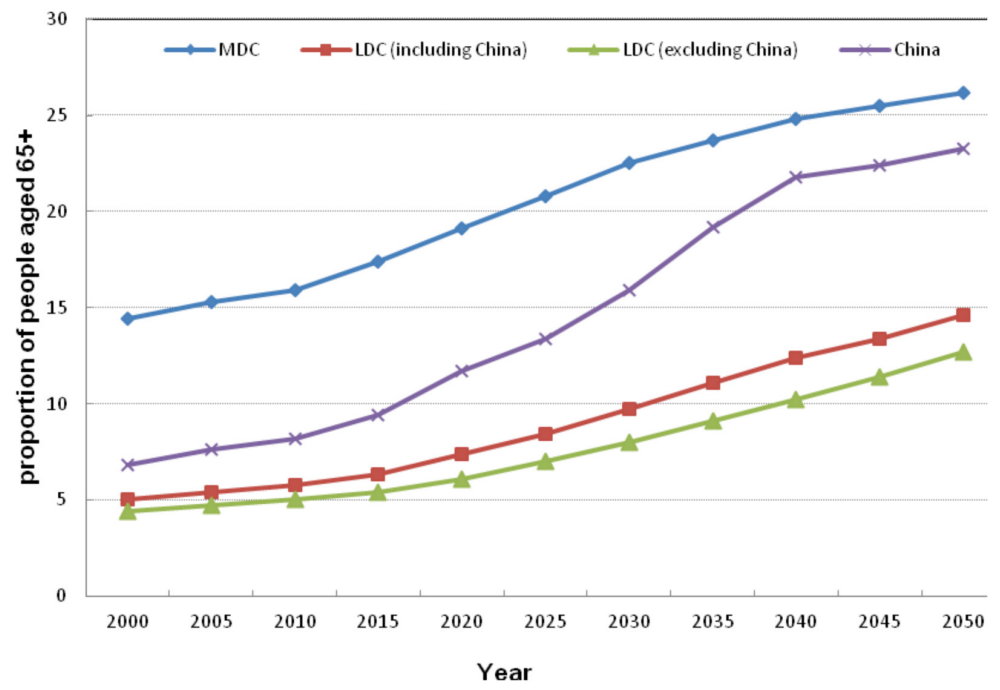


In contrast, future demographic changes will differ significantly in less developed countries. Their mortality and fertility will decline further. Because of the relatively high fertility and young age structure, their population will continue to grow; it will be close to 8 billion and account for 86 per cent of the world total by 2050 . While the increase in the proportion of old people will accelerate, their dependency ratio will stay at or be slightly lower than the current level owing to the decrease in the child dependency ratio, as indicated by Figures 16.5 and 16.6. Many less developed countries will benefit from this change. Furthermore, many of these countries will experience rapid urbanisation. Largescale rural-to-urban migration is likely to accompany their future economic growth.

Of course, countries regarded as 'less developed' according to the UN classification are very different both in their levels of economic development and in their stages of demographic transition. Such variations are likely to remain in the foreseeable future. While some of these countries are now in many ways closer to more developed countries than to other less developed countries, the above discussion and major conclusions drawn from it are still useful in providing a brief summary of world demographic changes over the past half-century and their major trends in the near future.

\section{The major characteristics of China's future demographic changes}

What are China's current demographic situation and future population trends, and how do they differ in comparison with those in other parts of the world? Before answering these questions, it is useful to briefly comment on a number of characteristics of demographic change that are likely to take place in China in the next few decades. In comparison with socioeconomic developments that are affected by many factors and thus often difficult to predict, China's future demographic changes have the following notable differences.

Population changes are determined by a few demographic factors and China's future population changes are no exception. If we consider only changes in population size and structure, they are determined exclusively by fertility, mortality and migration. Since China is a large country, the influence of international migration on population growth is rather small. Although internal migration could greatly change its spatial distribution, it has no impact on the size and structure of the national population.

China's future mortality and fertility changes are likely to be relatively slow and steady rather than dramatic. China had a rapid mortality decline in the 1950s and 1960s, and a great fertility reduction in the 1970s. But changes of this kind are unlikely in the next one or two decades. Unless catastrophic infectious diseases, natural disasters or wars strike, China's mortality will decline steadily or stay at a low level. Similarly, there might be some small fluctuations in fertility, but a drastic increase or decrease is unlikely in the near future.

It is also expected that China's future mortality and fertility changes are likely to lack 'elasticity'. Future mortality and fertility trends-which differ from certain economic phenomena or activities that can be stimulated or altered relatively easily by government financial, monetary or taxation policies - are less likely to change drastically as a response to government policies or interventions of other kinds. China's nationwide family-planning campaign did contribute to its rapid fertility reduction in the 1970s. But now the major 
demographic challenge is no longer to control birth. Until now, there has been no evidence suggesting that moderate incentive policies have brought back sustained replacement fertility in countries where it had fallen to a very low level.

Population momentum will also affect future demographic changes. Although China's fertility has been below replacement since the early 1990s, China's population has grown for the past 20 years, and this trend will continue for at least another decade, because of the influence of the population age structure. Similarly, when China's population size starts falling, the trend might not stop quickly, even if fertility could be raised to a level that is moderately higher than that of replacement. Furthermore, even if effective policies or interventions were available to increase fertility, the fertility adjustment might not contribute positively to economic development until some 20 years later.

For the reasons summarised above, China's future demographic changes are more predictable than its socioeconomic development. Regardless of what we think about their importance and consequences, China's major population trends have already formed a demographic backdrop for, and will exert a significant impact on, its socioeconomic development in the next quarter of a century.

\section{China's future demographic trends}

So what kind of demographic changes will take place in China in the next few decades? First is the change in the national population. According to the United Nations' 2008 medium variant population projection, China's national population was about 1.35 billion in 2010. ${ }^{4}$ It will reach 1.46 billion in 2030 and then start to decline. By 2050, the Chinese population will fall to 1.42 billion (UN 2009). ${ }^{5}$ While China's population will continue to grow for another 15 to 20 years, it will lose the title of the most-populous country in the world to India in the mid to late 2020s. Moreover, the magnitude of China's future population increase will be much smaller than that recorded in the past half a century. The nature and major characteristics of the increase will also differ greatly from those observed in the 1960s and 1970s. Current population growth is the result of the population momentum created by past demographic changes. Under the surface of such an increase, a negative intrinsic growth rate has already been recorded. ${ }^{6}$ Accordingly, despite the fact that continuing population growth can still put some pressure on various kinds of resources and economic development, it is no longer a major demographic concern for future long-term development.

Second, similar changes will take place in the working-age population (those aged 1564). According to the United Nations' 2008 medium variant population projection, China's working-age population will reach its peak during the period 2010-15, at approximately 1 billion. After that it will begin to fall and decrease by 130 million by 2050 . The decline in the proportion of the working-age population will start earlier - falling from the current 72 per cent to 61 per cent in 2050. Since most Chinese, and those in urban areas in particular, will retire at or before age 60 , changes in the number of those aged 15-59 might be more relevant to many policy issues. According to the United Nations' 2008 projection, the proportion of people aged 15-59 reached 68 per cent in 2010, and it will now start to fall. ${ }^{7}$ By 2050, its size 
will decrease by 160 million. The decline of the younger working-age population (15-24) is particularly notable - by more than 80 million or 36 per cent in the next 40 years. As a result, China's working-age population will become older (UN 2009).

The third major demographic trend is that China's fertility is very likely to stay below or far below replacement in the near future, which will have a profound impact on longterm demographic and socioeconomic development. Rising fertility can prevent the Chinese population from decline, and indeed some scholars have recently argued that the Chinese Government should change its fertility policy. It is important, however, to be aware of the fact that China's current low fertility is no longer a result of, or caused mainly by, the restrictive family-planning policies, although they are still in place. Evidence shows that major changes in people's fertility desires and reproductive culture have already taken place in China. Young couples increasingly want to have a small family or have no children at all (Cai 2010; Gu and Wang 2009). These changes have largely conformed to the fertility trends observed in many countries and are similar to those recorded in several East Asian populations in recent decades. It is also important to note that in comparison with many other countries, in China, age at marriage is relatively low, the proportion of nevermarried people is still high, and the proportion of those never having children remains at a very low level. All of these are, however, likely to change in the near future. Increasing celibacy, postponing marriage to a later age and a rising proportion of childless people could soon become non-trivial factors contributing to China's low fertility. China's intrinsic population growth rate had already become negative in the early 1990s because of its belowreplacement fertility. Under the conditions used in the United Nations' 2008 population projection, China's population is expected to experience a long-term decline from the mid or late 2020s. The decline will continue in the rest of this century and could well last into the next century.

China's fourth major demographic trend is rapid population ageing. Figure 16.5 shows that China's current dependency ratio is only 39-perhaps the lowest level ever recorded. It will increase slightly thereafter, but its level will remain lower than that before 1985 and also lower than that in more developed countries. China can continue to benefit from its demographic dividend in the next quarter of a century. China's aged population and old-age dependency ratio will, however, increase rapidly. According to the United Nations' 2008 population projection, the number of people aged 65 and older was 111 million in $2010 .^{8}$ In the next 40 years, that number will further increase, and reach 331 million by 2050. Their proportion will rise from 8 per cent to 23 per cent, as shown in Figure 16.6. The number of people aged 80 and over will grow even faster. By 2050, the number of people in this age group will increase from the current less than 20 million to more than 100 million. As a result of these changes, the median age of the Chinese population will increase from 34 years in 2010 to 45 years in 2050 (UN 2009). As far as population ageing is concerned, China will become very similar to the industrialised countries.

The fifth major demographic trend is the increase in population movement and urbanisation. Differing from many other countries, in China, those who have left their homes and work in other cities or areas are often referred to as the 'floating population'. Many of these migrants, including their family members, are not given the same rights as the local 
people. Largely for these reasons, they tend to be less stable in terms of residence and employment, and thus often travel between the place where their permanent registration is held and the place where they work. Because of the difficulties in defining the urban and migrant populations and the difficulties in registering the floating population and monitoring its movements, there have been considerable uncertainties in determining the size of China's urban and rural populations and their future changes. There is, however, a general consensus among Chinese authorities and academics that China's internal migration has now reached an unprecedented scale and the speed of urbanisation will increase. According to the preliminary results of the 2010 census, China's floating population has reached 221 million and almost half of the population has lived in cities and towns (NBS 2011). Even according to UN projections, which give a lower figure, China's urban population was more than 600 million or 47 per cent of the national population in 2010, and it is likely to rise to more than 1 billion or 73 per cent of the total population by 2050 (UN 2010).

\section{China's future sustainable development and major demographic challenges}

Most of the demographic changes discussed above will take place in the next few decades, which will significantly affect Chinese society. From a demographic viewpoint, to create and maintain sustainable, generally favourable and relatively stable demographic conditions is one of the most important strategies for promoting future development.

A key step for the Government to facilitate and maintain such a developmentfriendly demographic environment is to closely monitor demographic changes and reduce preventable negative demographic impacts (such as great fluctuations in population size and structure and extremely low fertility) in a timely fashion through effective guidance and interventions. While future demographic changes are relatively easy to project, reliable projection results cannot be produced without accurate data about the current state of the population. One urgent issue in this respect is to resolve the puzzle surrounding China's recent fertility level and population size.

The Chinese Government reports that the country's TFR has been about 1.8 since the mid1990s. It also insists that this level will be maintained in the next three decades (National Strategy on Population Development Research Group 2007). Most demographers, however, believe that China's TFR was lower than 1.8 in 1995 and probably about 1.6 in 2000. It has been even lower and perhaps about 1.5 since then. They also suggest that China's TFR might stay at a level lower than 1.8 in the near future (Cai 2008, 2010; Gu and Wang 2009; Guo and Chen 2007; Retherford et al. 2005; Scharping 2005; Zhang and Zhao 2006; Zhao and Guo 2010; Zhao and Zhang 2010). Because of that, there are notable differences among various estimates of China's current and future population sizes. For example, according to The General Report on China's National Strategy on Population Development, China's national population, which is currently close to 1.35 billion, will grow to 1.43 billion by 2020 and reach a peak of about 1.5 billion in 2033 (National Strategy on Population Development Research Group 2007:36). These results are largely similar to those produced by the United Nations' 2008 medium variant population projection. They differ significantly, however, from the preliminary results of the United Nations' 2010 population projection and those projected recently by the US Census Bureau, which were based on lower but more reliable 
fertility estimates (UN 2009, 2011; US Census Bureau 2011). According to the United Nations' latest population projection, China's current population is about 1.34 billion and will reach the peak of approximately 1.4 billion in 2025. In 2033, China's population size will be about 1.39 billion, or nearly 100 million smaller than that suggested by the Chinese Government. For the year 2050, the preliminary result of the United Nations' 2010 population projection is also 120 million smaller than that obtained from the 2008 projection (UN 2009, 2011). Differences of nearly 20 per cent in estimated fertility levels and of about 100 million people in projected population size are by no means negligible. They will have significant impacts on the formation of China's future development plans and strategies. To clear up the confusion created by these uncertainties, great effort must be made to improve China's demographic data collection and data quality.

It is a pity that the figures reported in this chapter could not be based on the final results of the United Nations' 2010 population projection because they are still not available. ${ }^{9}$ If these results were used, the decline in China's national population and working-age population would be earlier and of greater magnitude, the increase in the proportion of old people and the old-age dependency ratio would be faster and larger, and the momentum of population decline would last longer than that suggested by the United Nations' 2008 population projection results. Their consequences would therefore be more severe. For these reasons, China needs to carefully consider the negative and long-term impact of very low fertility and prepare for effective policy intervention accordingly.

In the twenty-first century - at least the first half of the century - one of China's most significant demographic changes is the increasing population ageing. It is important to recognise that population ageing, in China and elsewhere, is largely the product of two demographic successes: first, effectively eradicating premature deaths and improving longevity; and second, effectively controlling reproduction or avoiding unwanted pregnancies. Because mortality will continue to decline and fertility will probably stay at a relatively low level, population ageing is likely to be an irreversible trend of longterm development rather than a historical event occurring at a particular time. Hence, it is more meaningful to think about how to prepare ourselves to live in this new demographic environment than to talk about preventing or delaying population ageing, although the latter might not be completely meaningless within a short period or for a small population. As demographic successes, improving longevity and population ageing have brought considerable benefits to humankind and our society. In addition to the demographic dividend that has been addressed by many scholars (Mason and Tomoko 2004; Wang and Mason 2007), these changes provide an opportunity for people to increase the number of years that they live in a healthy state. Although the findings are not conclusive, many studies have shown that along with improving longevity, the proportion of healthy life expectancy out of the total life expectancy has increased. People can now spend a greater proportion of their life in production or other types of creative activities. This in turn can help to overcome the difficulty caused by rapid population ageing. In the long run, difficulties brought about by this change cannot be dealt with by promoting high (above replacement) fertility or migration. They can be overcome only by rapidly developing the economy, adjusting related social and economic policies, and establishing the facilities and social institutions that will be required by the increasingly ageing society. 
Another major challenge facing China is the significant increase in urbanisation and migration. By international standards, China's current level of urbanisation is not high, but the scale of its rural-to-urban migration (though it might be less permanent) and urbanisation is gigantic. Whether China can successfully manage its future urbanisation is a serious challenge in maintaining its development momentum and in building a truly harmonious society. For a very long time, China's development policies have been urban centred and rural surplus workers have been used as a reservoir to adjust the labour supply for urban development. Rural-to-urban migrants have rarely been given rights equal to those their urban counterparts have, but often have been blamed for many problems that have occurred in the process of urbanisation. This not only prevents China from smoothly advancing its urbanisation, it could potentially become a major source of social disturbance and instability. Largely due to the floating nature of China's current migrant population and the impact of China's long-established two-tier urban and rural development and social policies, China's future urbanisation and rural-to-urban migration are not only a demographic or economic process. They are, in many ways, a profound demographic, social, economic and political transformation.

\section{Conclusions}

The worldwide demographic transition has been one of the most significant events in recent history and has played a crucial part in the creation of the modern world. While China's demographic transition started later in comparison with more developed countries, it has been faster. As a result of the rapid mortality and fertility decline, China's life expectancy at birth has now reached 74 years, its TFR has fallen well below replacement, and the dependency ratio has decreased to a very low level. China's recent economic development has benefited greatly from such favourable demographic conditions. China's demographic changes will not stop here, however. After its unprecedented growth in the second half of the twentieth century, China's national population-driven by its fertility reduction, especially the below-replacement fertility level in the past two decades - will soon begin a long-term decline. The decline in its working-age population will start earlier. Because of that and the rapid population ageing, China's dependency ratio will rise. In comparison with recent years, in the future China's demographic conditions will become less favourable to its economic growth. In addition, China will face the great challenge of large-scale rural-to-urban migration and rapid urbanisation. To meet these challenges, China needs to form more realistic strategies to create and maintain sustainable, generally favourable and relatively stable demographic conditions, which are of great importance to the country's future prosperity and long-term development.

\section{Acknowledgment}

The author would like to thank Jiaying Zhao for her assistance in drawing all the graphs used in this chapter. The author also acknowledges the partial support provided by an Australian Research Council Research Grant (DP1096696). 


\section{Bibliography}

Banister, J. 1987, China's Changing Population, Stanford University Press, California.

Barclay, G. W., Coale, A. J., Stoto, M. A. and Trussell, T. J. 1976, 'A reassessment of the demography of traditional rural China', Population Index, vol. 42, no. 4, pp. 606-35.

Cai, Y. 2008, 'An assessment of China's fertility level using the variable- $r$ method', Demography, vol. 45, no. 2, pp. 271-81.

Cai, Y. 2010, 'Social forces behind China's below replacement fertility: government policy or socioeconomic development', Population and Development Review, vol. 36, no. 3, pp. 419-40.

Caldwell, J. 1986, 'Routes to low mortality in poor countries', Population and Development Review, vol. 12, no. 2, pp. 171-219.

Campbell, C. 2001, 'Mortality change and the epidemiological transition in Beijing, 16441990', in T. Liu, J. Lee, D. S. Reher, O. Saito and F. Wang (eds), Asian Population History, Oxford University Press, Oxford.

Dyson, T. 2010, Population and Development: The demographic transition, Zed Books, London.

Gu, B. and Wang, F. (eds) 2009, An Experiment of Eight Million People, [in Chinese], Social Sciences Academic Press, Beijing.

Guo, Z. and Chen W. 2007, 'Below replacement fertility in Mainland China', in Z. Zhao and F. Guo (eds), Transition and Challenge: China's population at the beginning of the 21st century, Oxford University Press, New York.

Huang, R. and Liu, Y. 1995, Mortality Data of China Population, [in Chinese], Data User Service, China Population Information and Research Center, Beijing.

Lavely, W. and Freedman, R. 1990, 'The origins of the Chinese fertility decline', Demography, vol. 27, pp. 357-67.

Livi-Bacci, M. 2007, A Concise History of World Population, (Fourth edition), Blackwell Publishing, Oxford.

Mason, A. and Tomoko, K. 2004, East Asian economic development: two demographic dividends, Economics Series Working Papers No. 83, East-West Center, Honolulu.

Meslé, F. 2004, 'Mortality in Central and Eastern Europe: long-term trends and recent upturns', Demographic Research (Special Collection 2), pp. 45-70.

National Bureau of Statistics (NBS) 2011, The Sixth National Census, Bulletin No. 1, China Statistics Press, Beijing, viewed April 2011, <http://www.stats.gov.cn/tjfx/jdfx/ t20110428_402722253.htm>

National Strategy on Population Development Research Group 2007, The General Report on China's National Strategy on Population Development, [in Chinese], China Population Publishing House, Beijing. 
Ren, Q., You, Y., Zheng, X., Song, X. and Chen, G. 2004, 'The levels, patterns and regional variations in mortality of China since the 1980s', [in Chinese], Chinese Journal of Population Science, no. 3, pp. 19-29.

Retherford, R. D, Choe, M. K., Chen, J., Li, X. and Cui, H. 2005, 'Fertility in China: how much has it really declined?', Population and Development Review, vol. 19, no. 1, pp. 57-84.

Scharping, T. 2005, 'Chinese fertility trends 1979-2000: a comparative analysis of birth numbers and school data', [in Chinese], Population Research, no. 4, pp. 1-15.

United Nations (UN) 2009, World Population Prospects: The 2008 revision, United Nations, New York.

United Nations (UN) 2010, World Urbanization Prospects: The 2009 revision, United Nations, New York.

United Nations (UN) 2011, World Population Prospects: The 2010 Revision, United Nations, New York.

US Census Bureau 2011, Preliminary Results for World Population Prospects 2010, Population Division, US Census Bureau, Washington, DC, viewed March 2011, <http://www.census. gov/ipc/www/idb/country.php>

Wang, F. and Mason, A. 2007, 'Population ageing: challenges, opportunities, and institutions', in Z. Zhao and F. Guo (eds), Transition and Challenge, Oxford University Press, Oxford.

Yao, X. 1995, Fertility Data of China, [in Chinese], China Population Publishing House, Beijing.

Zhang, G. and Zhao, Z. 2006, 'Re-examining China's fertility puzzle: data collection and quality over the last two decades', Population and Development Review, vol. 32, no. 2, pp. 293-321.

Zhao, Z. 1997, 'Demographic systems in historic China: some new findings from recent research', Journal of the Australian Population Association, vol. 14, no. 2, pp. 201-32.

Zhao, Z. and Guo, F. 2007, 'Introduction', in Z. Zhao and F. Guo (eds), Transition and Challenge: China's population at the beginning of the 21st century, Oxford University Press, New York.

Zhao, Z. and Guo, Z. 2010, 'China's below replacement fertility: a further exploration', Canadian Studies in Population, vol. 37, pp. 525-62.

Zhao, Z. and Zhang, X. 2010, 'China's recent fertility decline: evidence from reconstructed fertility statistics', Population, vol. 65, no. 3, pp. 451-78.

Zheng, Z., Cai, Y., Wang, F. and Gu, B. 2009, 'Below-replacement fertility and childbearing intention in Jiangsu Province, China', Asian Population Studies, vol. 5, no. 3, pp. 329-47. 


\section{Endnotes}

1. According to the Population Information and Research Centre of China, life expectancy at birth for the Chinese population increased to 56 in 1957, 64 in the early 1970s, and 68 in 1981 (Huang and Liu 1995).

2. As shown by the preliminary results of the United Nations' 2010 projection, lower fertility estimates have been used in this round of population projection, which will be further discussed later.

3. Since the final results of the United Nations' 2010 population projection are not available, a large part of the discussion has been based on the United Nations' 2008 medium variant population projection results. Because the UN Population Division is very likely to base its 2010 final projection on lower fertility estimates, the results will be different from those obtained from the 2008 projection.

4. According to China's 2010 census, its national population (excluding the population of Hong Kong, Macau and Taiwan) was 1.34 billion (NBS 2011).

5. These results are most likely to have overestimated China's future population growth and this will be further discussed in the next section.

6. Changes in a population can be examined in two ways. First, the increase or decrease of the total population can be examined. Second, whether a generation can reproduce itself - which is often measured by whether a group of women can produce enough daughters to replace themselves - can be examined. Because of the impact of age structure, changes in the second type of population growth - which is called intrinsic population growth - might not be the same as those in the first type of population growth.

7. According to China's recent census, the proportion of people aged 15-59 was slightly higher-at 70 per cent-in 2010 (NBS 2011).

8. This figure is about 7 million lower than that obtained from China's 2010 census (NBS 2011).

9. An alternative is to base the study on China's 2010 census data, but the detailed results are still not available. The choice of using the United Nations' 2008 medium variant population projection results rather than making a new projection also arises from the consideration of ensuring the consistency in comparing demographic changes between China, more developed countries and less developed countries. 